\title{
Towards Effective Patient Simulators
}

\author{
Vadim Liventsev ${ }^{1,2 *}$, Aki Härmä ${ }^{2}$ and Milan Petković ${ }^{1,2}$ \\ ${ }^{1}$ Eindhoven University of Technology, Eindhoven, Netherlands, ${ }^{2}$ Philips Research (Netherlands), Eindhoven, Netherlands
}

In this paper we give an overview of the field of patient simulators and provide qualitative and quantitative comparison of different modeling and simulation approaches. Simulators can be used to train human caregivers but also to develop and optimize algorithms for clinical decision support applications and test and validate interventions. In this paper we introduce three novel patient simulators with different levels of representational accuracy: HeartPole, a simplistic transparent rule-based system, GraphSim, a graph-based model trained on intensive care data, and Auto-ALS - an adjusted version of an educational software package used for training junior healthcare professionals. We provide a qualitative and quantitative comparison of the previously existing as well as proposed simulators.

Keywords: reinforcemenet learning, healthcare, simulators and models, clinical methods, markov decision chain

\section{INTRODUCTION}

\section{OPEN ACCESS}

Edited by:

Naimul Khan,

Ryerson University, Canada

Reviewed by:

Batyrkhan Omarov,

Al-Farabi Kazakh National University, Kazakhstan

Arriel Benis,

Holon Institute of Technology, Israel

${ }^{*}$ Correspondence:

Vadim Liventsev

v.liventsev@tue.nl

Specialty section: This article was submitted to Medicine and Public Health, a section of the journal Frontiers in Artificial Intelligence

Received: 20 October 2021 Accepted: 29 November 2021 Published: 15 December 2021

Citation:

Liventsev V, Härmä A and Petković $M$ (2021) Towards Effective Patient Simulators.

Front. Artif. Intell. 4:798659. doi: 10.3389/frai.2021.798659
Patient simulators have been applied extensively in nursing education (Wendy et al., 2001; Nehring and Lashley, 2004; McFetrich, 2006), both as physical mannequins and as digital patient simulation software (Lee et al., 2020). However, patient simulators have another important application: they can be used to predict how patients' health would respond to various treatments and thus evaluate proposals for novel treatment strategies. And now, with the advent of artificial intelligence, one can use reinforcement learning (Yu et al., 2019), genetic programming (Koza, 1994; Niazkar and Niazkar, 2020) or both (Liventsev et al., 2021) to generate treatment strategies that lead to the best outcomes for the patient according to a certain simulator. In this paper we review the field of interactive patient simulators and evaluate their potential to be used for automated treatment discovery.

The contributions of this paper are as follows:

- A systematic review of patient simulators in Healthcare Informatics

- Auto-ALS - a learning aid for junior healthcare professionals adapted to reinforcement learning

- HeartPole-a novel simple and transparent pseudosimulator designed to be a convenient benchmark

- GraphSim-a data-driven graph-based simulator based on MIMIC-IV dataset (Johnson et al., 2021) for maximal accuracy

\subsection{Scope of This Survey}

This survey only includes interactive patient simulators where a (human or software) agent communicates its clinical intervention decisions to the simulator and the simulator, in turn,

1) Predicts future patient state given current patient state and clinical interventions.

2) Rewards or punishes (negatively rewards) that judges if the result of the treatment is positive or negative. The model can be as simple as just negatively rewarding patient deaths or as complex as using the predictions of the prediction model to help the agent recognize during the episode whether they are on the right track to a positive outcome. 
Any interactive simulator with a prediction model and a reward model (and thus, all simulators in this paper) can be represented as Episodic Partially Observable Markov Decision Process (EPOMDP) as described in (Liventsev et al., 2021):

$M=\left(\mathcal{S}_{n t}, \mathcal{S}_{t}, \mathcal{A}, \mathcal{O}, p_{o}(o \mid s, a), p_{s}\left(s_{\text {next }} \mid s_{\text {prev }}, a\right), p_{r}(r \mid s, a), p_{\text {init }}(s)\right)$

Here, $\mathcal{S}_{n t}$ is the set of non-terminal patient states. $\mathcal{S}_{t}$ is the set of terminal patient states (death, discharge from hospital or end of outpatient treatment) such that getting to one of these states signifies the end of an episode. $\mathcal{A}$ is the set of actions that the learning agent can perform, and $\mathcal{O}$ is the set of observations about the current state that the agent can make. Distributions $p_{o}, p_{s}$, and $p_{\text {init }}$ represent the simulator's prediction model, while $p_{r}$ represents the reward model.

Markov Decision Processes are a standard formalism in the field of Reinforcement Learning (Sutton and Barto, 2018), so standard RL algorithms can be used in conjunction with any simulator from this paper for treatment discovery.

There are 2 notable examples of systems that can be referred to as patient simulators, but do not make it easy to evaluate treatment strategies and fall outside the scope of this survey:

1) prediction models without a reward model

2) synthetic data generators (Chen et al., 2021)

They former are tools like HumMod (Hester et al., 2011) that help predict how change in one variable of patient health can affect another, for instance, how digoxin intake affects blood pressure. A reward model is absolutely necessary for the application area that we focus on in this paper-development and validation of treatment strategies. Developing it is also a nontrivial problem in its own right-which health outcomes are considered good depends on severity of the patient's condition (alive is a sufficiently successful outcome for the most severe conditions, but not for others) as well as the patient's own preferences (Street et al., 2012; MühlbacherMühlbacher and Juhnke, 2013). Thus, we view prediction models as components that can be used to develop a full simulator in the future.

The latter simulate clinical scenarios by generating samples of clinical histories. They provide a privacy-preserving way to generate a lot of training data for treatment discovery (James et al., 2018; Gong et al., 2020; Rankin et al., 2020; Wang et al., 2021), however, they don't model counterfactuals. That is, they don't answer questions of the sort "How would the patient's health respond to decision X"? For example, Synthea (Cavalcante et al., 2016) is a framework for generation of entire synthetic Electronic Health Records, EHRs, of patients, from the first to the last patient encounter, but the system does not provide ways to test alternative interventions on patients.

\subsection{Simulators as Models}

What makes an effective patient simulator? We argue that there are two use cases for a patient simulator: modeling and benchmarking, with divergent effectiveness criteria.
Firstly, a simulator can be used as a patient model aiming to represent the real world evolution of patient health in response to various treatments as closely as possible. The effectiveness criterion for a patient model is simple-accuracy. Accuracy can be measured by comparing the simulator's predictions against a dataset of clinical histories. One metric in particular that has been used for simulator validation is mean predictive error over dynamic transitions (Summers et al., 2009). If the simulator was obtained with machine learning, the data also has to be different from the training data: a different dataset or a holdout set can be used for that purpose.

The trust graph (see Figure 1) for a model is equally simple: if the data used for development of the simulator is a representative sample of the true distribution of the patients and the methodology used to turn build an interactive simulator based on these data is sound, the simulator is an accurate representation of a real world patient. And if the training algorithm used to discover the optimal treatment under this simulator is sound, the resulting treatment strategy can be trusted as well. Unfortunately, these conditions often hold only partially, or do not hold at all.

\subsection{Common Biases}

One type of bias, present in most data-driven healthcare simulators is sampling bias. If patient data is used to develop a simulator, this data is a sample (and, potentially, a biased sample) of the patient population that may or may not represent the population accurately. This problem is exacerbated by a profound shortage of healthcare datasets available to the research community. The biggest currently available dataset is MIMICIV (Johnson et al., 2021) - a large database of electronic health records in intensive care. However, MIMIC-IV was collected in one hospital in Boston and thus represents a demographically biased sample of the population. This bias is likely to percolate into any simulator developed based on MIMIC-IV.

But the most crippling type of simulator bias that makes many simulators completely unsuitable to serve as patient models is confirmation bias. Confirmation bias occurs when the developers of a simulator are aware of state of the art clinical practices and, intentionally or unintentionally, develop a simulator that rewards them and punishes alternatives. In didactic simulators used for education of clinical professionals, confirmation bias exists by design: students are, after all, trained to follow established clinical protocols. For a particular example of intentional confirmation bias in simulator design see Virtu-ALS (section 2.3). In this simulator, a decision that violates the existing emergence care protocol will be registered as a mistake, explained as such to the student, and not actually implemented.

\subsection{Simulators as Benchmarks}

Heavily biased and otherwise inaccurate simulators should not be used as patient models and should not be used as a basis for treatment recommendations. However, they can make a great benchmarks for learning algorithms. A benchmark is used not to develop a novel treatment strategy and apply it in the real world, but to compare different machine learning algorithms for healthcare against each other. Simulators that exhibit confirmation bias are a good fit for this, since they are similar 


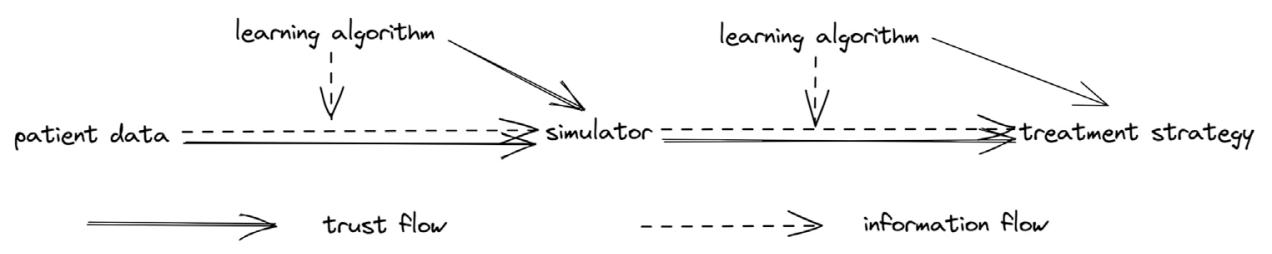

FIGURE 1 | Trust graph of simulator as a model.

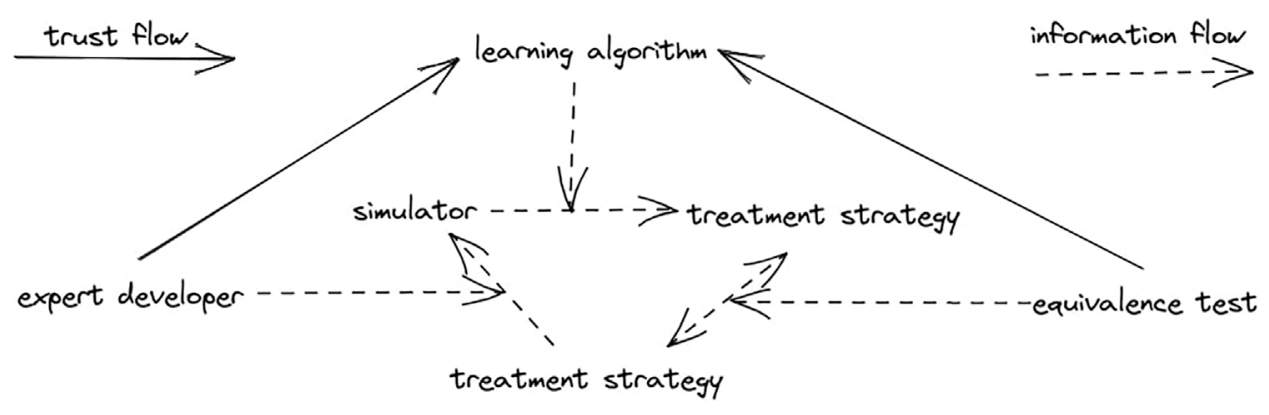

FIGURE 2 | Trust graph of simulator as a benchmark.

to true patient models, but unlike in a true patient model, treatments that these simulators evaluate as optimal are known beforehand. One can test a learning algorithm by applying it to a biased simulator and checking that the resulting strategy is equivalent to the strategy that simulator developers had in mind, as displayed on the trust graph in Figure 2 .

A crucial metric for effective benchmarks is their difficulty. A difficult simulator is one where most learning algorithms fail to discover an effective treatment strategy, i.e. a strategy that leads to positive health outcomes. A benchmarks's purpose is separating stronger learning algorithms from weaker ones, hence a good benchmark has to be not too difficult and not too easy. To get a grasp on simulator difficulty we train a baseline reinforcement learning model on each, see section 4.

Another desideratum for benchmarks is transparency. When a learning algorithm fails to discover an effective treatment strategy, it is very useful to understand what went wrong in detail. Any information output by the simulator other than its predictions as well as having easy access to its internal logic can be useful for developers.

Didactic simulators can be particularly good benchmarks (Liventsev, 2021a), but synthetic games akin to CartPole (Barto et al., 1983) and MountainCar (Moore, 1990) (commonly used as benchmarks for Reinforcement Learning algorithms) that aim to mimic only certain aspects of real life healthcare tasks can also be used. A particularly illustrative example is Healing MNIST (Krishnan et al., 2015) - a modified version of the industry standard MNIST Handwritten Digits (Li Deng, 2012) dataset to which rotations and random noise have been added. The authors argue that this dataset reflects important properties of healthcare tasks: rotations represent evolution of patient state over time, while the randomised "squares within the sequences are intended to be analogous to seasonal flu or other ailments that a patient could exhibit that are independent of the actions and which last several timesteps." Due to the data shortage in Healthcare many proposals for data-driven clinical decision support systems are tested on such relatively unrealistic benchmarks with the assumption that the system can be retrained on real patient data and simulators shall they arrive in the future.

\section{EXISTING SIMULATORS}

\subsection{Simglucose}

UVA/Padova (Man et al., 2014) is a set of equations used to model type 1 diabetes. The equations, outlined on Figure 3, were developed by clinical experts and validated on a dataset of 32 people aged $38 \pm 12$ years. It is widely used in Healthcare and even approved in the United States as a replacement for clinical trials. It provides $p_{o}(o \mid s, a)$ and $p_{s}\left(s_{\text {next }} \mid s_{\text {prev }}, a\right)$ (see section 1.1), so to be a full-fledged Markov Decision Process it only need $p_{r}(r \mid s, a)$. (Xie, 2018). solves exactly that by adding a reward function based on diabetes risk index as defined in (Clarke and Kovatchev, 2009) to the UVA/Padova simulator, providing a Reinforcement Learning environment for type 1 diabetes.

\subsection{GYMIC}

\subsubsection{Scope}

GYMIC (Kiani et al., 2019) is, unlike the previous examples, a fully data-driven simulator. It harnesses a subset of MIMIC (Johnson et al., 2021) dataset to address on one of the most challenging problems in emergency care-sepsis. The authors 


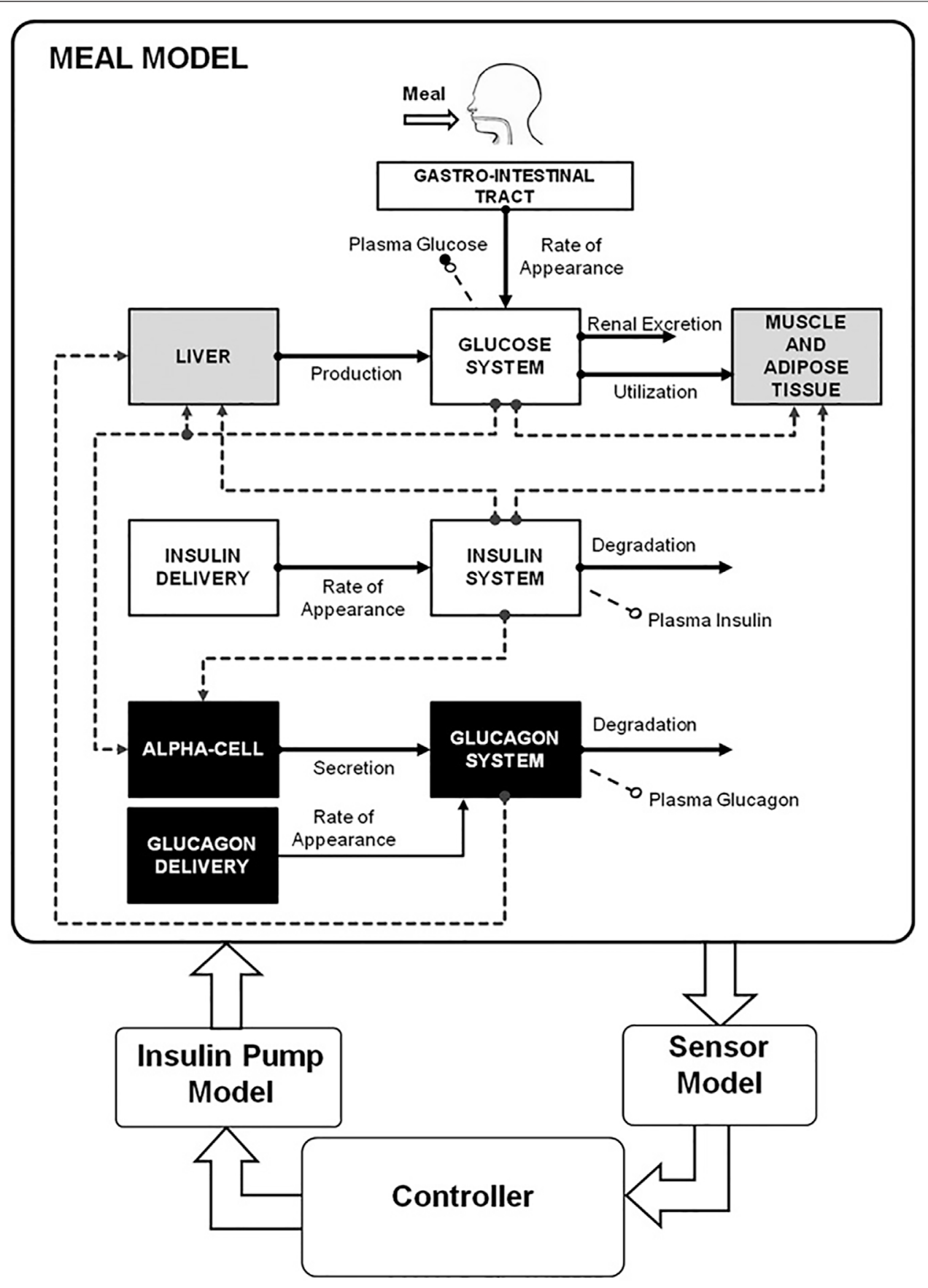

FIGURE 3 | UVA/PADOVA equations, visualised.

intentionally limit their scope to just sepsis in order to simplify the modelling task as well as because sepsis prevention has been identified as an area where doctors would particularly benefit from electronic decision support (Raghu et al., 2017; Walonoski et al., 2018).

\subsubsection{Prediction Model}

The prediction model of GYMIC simulator is defined as a solution to the following autoregression task:
1) A clinical history is a sequence of $(s, a)$ tuples

2) $a \in 0, \ldots 24$ is one of 25 possible vasopressor or intravenous fluid interventions-a cartensian product of five types of interventions and five dosage quantiles.

3) $s \in R^{46}$ is the patient's state at the moment this intervention was administered.

4) Predict the conditional state distribution $p_{s}\left(s_{t}, a_{t} \mid s_{t-1}\right.$, $\left.s_{t-2}, \ldots, s_{1}\right)$ 


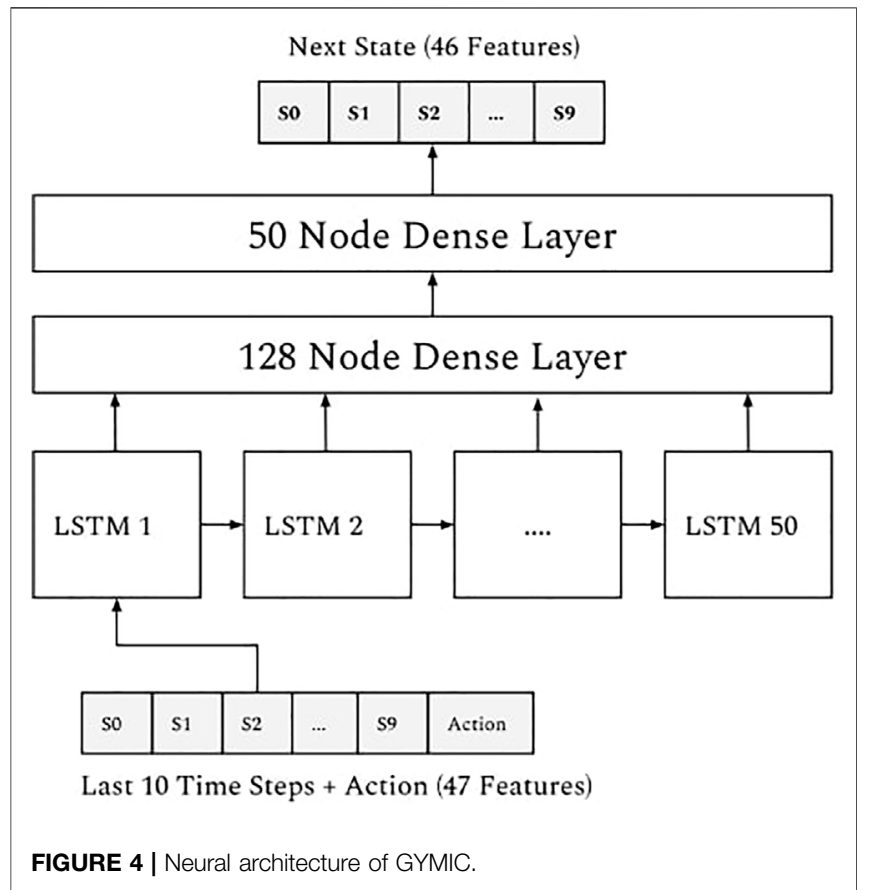

The dataset of clinical histories is produced by a preprocessing algorithm combining together all clinical records from MIMIC that relate to sepsis patients.

Autoregressive tasks of this nature arise in many fields like stock market prediction (Cavalcante et al., 2016; Jiang, 2021) or language modelling (Jozefowicz et al., 2016) where state of the art solutions can be found. The authors of GYMIC solve it with an LSTM (Hochreiter and Schmidhuber, 1997) neural network with two additional dense layers attached, see Figure 4 for the diagram. Faced with some of the mode collapse issues described in section 2.2.4 the authors also experimented with semi-supervised learning [? ]: they trained a variational autoencoder [?] on all patient states to replace the 46-vector representations of patient state $s$ with learned representations from the latent space of the VAE encoder $(s)$. The issues persisted.

\subsubsection{Reward Model}

Highlighting the gravity of contracting sepsis, GYMIC has only two outcomes: patient is discharged from intensive care or patient dies. Its reward model reflects that, giving the agent a large positive or negative reward at the end of the episode, depending on the outcome. However, in order to lower the difficulty of the simulator (delayed gratification makes training significantly harder (Mischel and EbbesenEbbesen, 1970; Gulwani et al., 2017)) an additional reward is provided during the episode, based on the evolution of the patient's SOFA score (Lambden et al., 2019) - a commonly used measure of sepsis severity:

$$
\begin{gathered}
r\left(s_{t}, s_{t+1}\right)=C_{0} 1\left(s_{t+1}^{\mathrm{SOFA}}=s_{t}^{\mathrm{SOFA}} \& s_{t+1}^{\mathrm{SOF}}>0\right)+ \\
+C_{1}\left(s_{t+1}^{\mathrm{SOFA}}-s_{t}^{\mathrm{SOFA}}\right)+C_{2} \tanh \left(s_{t+1}^{\mathrm{Lactate}}-s_{t}^{\mathrm{Lactate}}\right)
\end{gathered}
$$

A third reward component is proposed to negatively reinforce action severity and encourage the agent to use low doses of drugs an instance of confirmation bias as discussed in section 1.3, but a necessary step given the issues in section 2.2.4.

\subsubsection{Results and Issues}

Unfortunately, the experiments performed by the authors of GYMIC indicate extreme overfitting. Due to sampling bias and simply inadequate size of the dataset there are treatments that have only occurred a few times in the training data and have always resulted in a positive health outcome. In GYMIC these treatments are silver bullets that guarantee a successful outcome while in real life they are risky and potentially very harmful.

\subsection{Virtu-ALS}

Virtu-ALS is a didactic emergency care simulator mainly targeted at students and junior healthcare professionals, although its application as a reinforcement learning benchmark was anticipated and accounted for by the authors (Brisk et al., 2018). Its most prominent feature is its visual nature (Figure 5): the user has access to a 3D-rendered virtual copy of a hospital room, view the monitor, press buttons on a defibrillator, etc. However, the visual modality means that its observation space

$$
\mathcal{O} \subset R^{307200}
$$

Such a high dimensionality of the observation space makes it an extremely challenging reinforcement learning task. Tasks from this family have been solved with deep neural networks (Mnih et al., 2013), however not only does it require a long and expensive training process, it also means that resulting treatment strategies are black box neural networks that no clinical expert understands. This approach to decision making is extremely hard to introduce into clinical practice (Price, 2018; Watson et al., 2019).

Like most didactic simulators, Virtu-ALS exhibits considerable confirmation bias-any decision that's not supported by the standard emergency care protocol (American Heart Association Staff, 2006; Thim et al., 2012) is considered a mistake and rewarded negatively.

\section{PROPOSED SIMULATORS}

\subsection{Auto-ALS}

As our first model, we propose a low-dimensional version of Virtu-ALS. Auto-ALS is a modification of Virtu-ALS that removes all the complexity of dealing with a visual $3 \mathrm{D}$ environment while retaining all the complexity of dealing with a patient that requires emergency care. This is achieved by attaching an event listener to Virtu-ALS that registers all observable events that can occure in the simulator in response to the user's actions. The events are listed in Table 1, organized by which agent action can trigger which event. Tick is a special event that occurs every time the simulator is advanced a timestep, and is negatively reinforced, which when used with reinforcement learning algorithms discourages clinicaly unnecessary actions. 


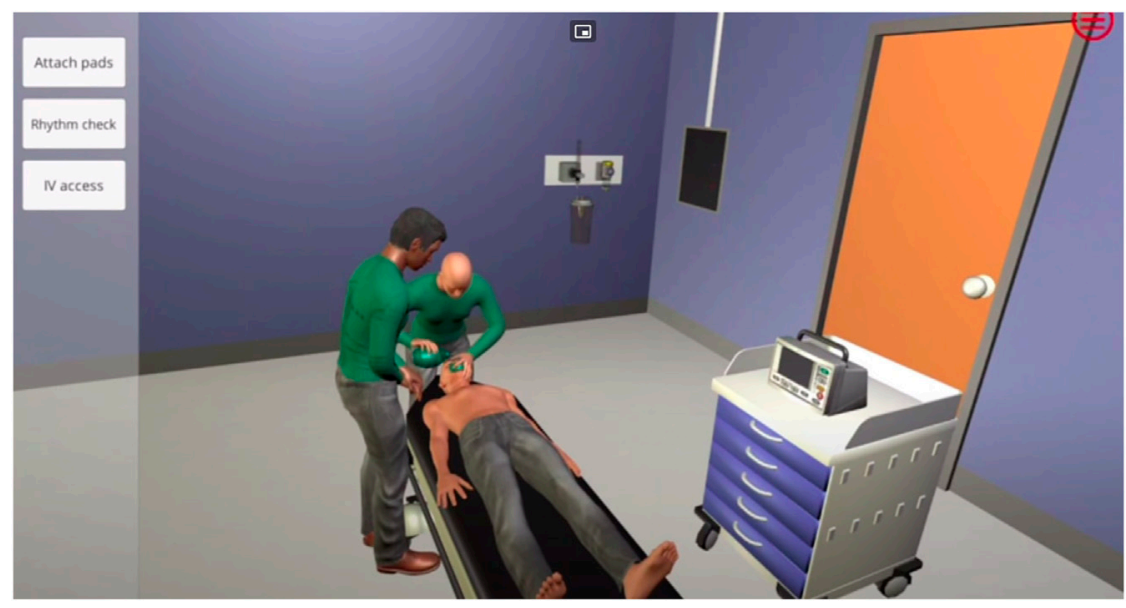

FIGURE 5 | Virtu-ALS.

TABLE 1 | All actions and observations of Auto-ALS.

\section{Agent actions}

AssessResponse

AssessAirway

AssessBreathing
Patient reactions

Rewards

ResponseVerbal, ResponseGroan, ResponseNone

0

BreathingNone, BreathingSnoring, BreathingSeeSaw,

BreathingEqualChestExpansion, BreathingBibasalCrepitations,

BreathingWheeze, BreathingCoarseCrepitationsAtBase,

BreathingPneumothoraxSymptoms, VentilationResistance,

MeasuredRespRate

RadialPulsePalpable, RadialPulseNonPalpable, MeasuredHeartRate

AVPU_A, AVPU_U, AVPU_V, PupilsPinpoint, PupilsNormal,

MeasuredCapillaryGlucose

ExposureRash, ExposurePeripherallyShutdown, ExposureStainedUnderwear,

MeasuredTemperature

HeartRhythm0, HeartRhythm1, HeartRhythm2, HeartRhythm3, HeartRhythm4,

MeasuredHeartRate, MeasuredMAP, MeasuredSats, MeasuredResps

Blunder

ABG, AirwayManoeuvres, GiveAtropine, GiveAdenosine, GiveAdrenaline,

GiveAmiodarone, GiveMidazolam, Venflon, Yankeur, DrawBloods,

BPCuffOn, BVM, Guedel, NRBMask, DefibOn, DefibAttachPads,

DefibShock, DefibCharge, DefibChangePaceCurrentDown,

DefibChangePaceCurrent, DefibEnergyDown, DefibEnergyUp,

DefibChangePaceRateDown, DefibChangePaceRateUp, DefibPace

Finish

$\begin{array}{lc}\text { Failure } & -1 \\ \text { Success } & 1 \\ \text { Tick } & r_{\text {tick }}\end{array}$

MeasuredHeartRate,

MeasuredRespRate, MeasuredCapillaryGlucose, MeasuredTemperature, MeasuredMAP, MeasuredSats, and MeasuredResps are measurements, events that have a value $(-\infty ;+\infty)$ associated with them.

The events in Table $\mathbf{1}$ only get registered if the agent has learnt some piece of information, meaning that, for example, AirwayVomit will only occur if the patient has vomit in their airway and the agent checked the airway (which is part of the standard protocol (Thim et al., 2012)). Assessment skills (knowing where to look and how to establish the patient's state) are crucial for patient resuscitation, hence revealing all known health variables to the agent would jeopardize the simulation.

The observation vector in Auto-ALS is based on all observations that have occurred between the beginning of the episode and current time. However, more recent observations are more likely to still be relevant and should be given priority. This is done with the following formula proposed in (Liventsev, 2021b):

$$
o^{+}=\left\langle o_{1} \in O_{1}, \exp \left(t_{1}-t\right), \ldots, o_{n} \in O_{n}, \exp \left(t_{n}-t\right),\right\rangle
$$




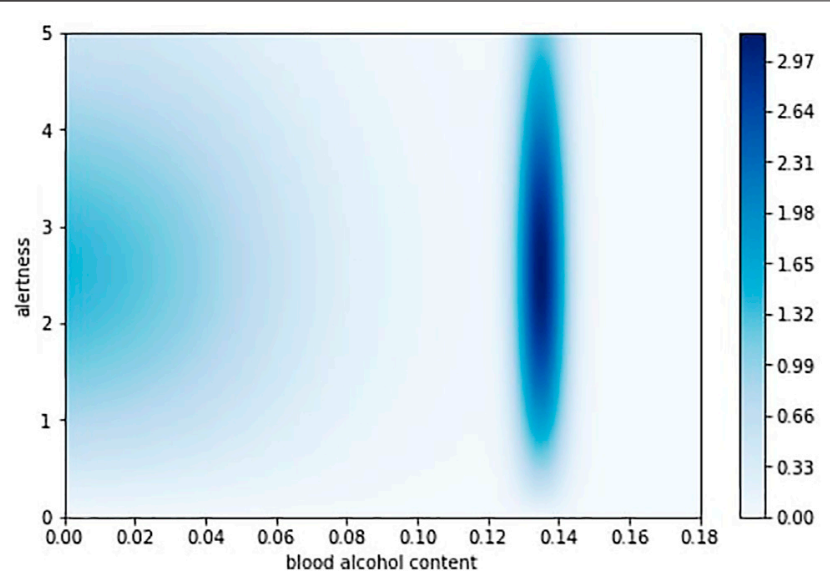

FIGURE 6 | Productivity function in HeartPole.

where $O_{i}$ is the value of the observation and $t$ is current time and $t_{i}$ is time when observation $i$ (for $i=5$, ResponseGroan) has last occurred and $\exp \left(t_{i}-t\right)$ represents its decaying relevance. For measurements, the $O_{i}$ equals the magnitude of the measurement, however, for binary obsevations $O_{i}$ would always be equal to one. For memory efficiency, for all $i$ that correspond to binary observations, $O_{i}$ is skipped from the $o^{+}$vector and the actual observation vector $o$ has size $36+7 * 2=50$, as opposed to $(36+7) * 2=86$.

See source code and documentation at (Liventsev, 2021c).

\subsection{HeartPole}

HeartPole focuses on simplicity and transparency at the expense of realism - it is based on a familiar scenario and a simple set of rules so that when a treatment performs badly it is easy to explore what exactly goes wrong. HeartPole simulates a creative professional trying to become more productive. However, many decisions that would help in the short term (not sleeping, consuming coffee and alcohol) can create long-term health issues that negate all short term gains.

In HeartPole state $s$ consists of alertness $s_{t}^{\text {alert }}$, hypertension $s^{\text {hypert }}$, intoxication $s^{\text {tox }}$ time since slept $s^{\text {tawake }}$, total time elapsed $s^{\text {ttotal }}$ and total work done $s^{\text {done }}$.

Over these parameters, we define productivity function $\eta\left(s_{t}^{\text {alert }}, s_{t}^{\text {tox }}\right)$ presented graphically on Figure $\mathbf{6}$ and heart attack probability $r\left(s_{t}^{\text {hypert }}\right)=\frac{\operatorname{sigmoid}\left(s_{t}^{\text {hypert }}\right)}{2}$. The agent receives small positive rewards for productivity and a very large negative reward if a heart attack occurs.

As shown on Figure 7, every half an hour awake, the agent observes $s_{t}$ and picks an action $a_{t}$ from discrete action space of just work, drink coffee (increases $s^{\text {alert }}$ and $s^{\text {hypert }}$ ), drink beer (decreases $s^{\text {alert }}$, increases $s^{\text {hypert }}$ and $s_{t}^{\text {tox }}$ ) and go to bed (sleep takes a lot of time, but reduces $s^{\text {hypert }}$ and $s_{t}^{\text {tox }}$ and without it alertness starts to fall very fast).

See source code and documentation at (Härmä et al., 2021).

\subsection{GraphSim}

Our last model is trained on MIMIC (Johnson et al., 2021) to maximise accuracy. MIMIC can be represented as a set of patients

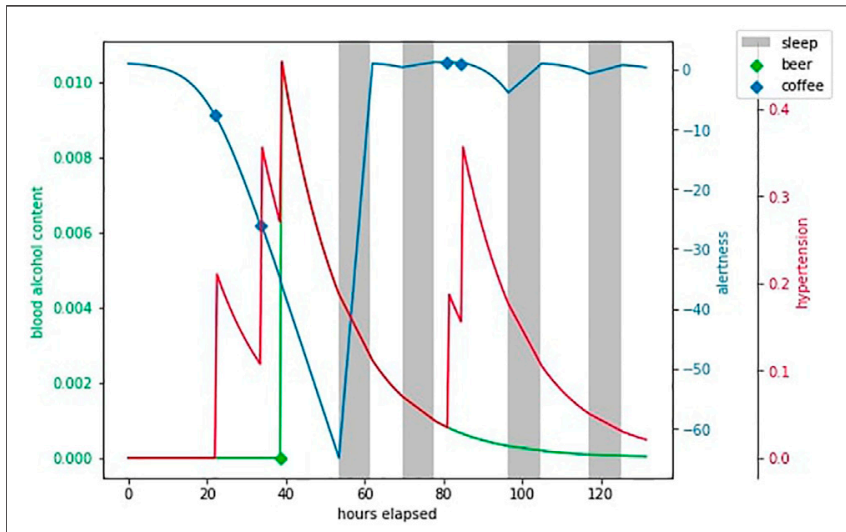

FIGURE 7 | An example episode in HeartPole.

where every patient is an oriented graph, its nodes are patient states, each state is a vector of various clinical measurements, such as blood pressure and oxygen saturation, whereas arcs that connect the patient states are doctors actions, each action a vector of administered drug doses. These oriented graphs can also be viewed as disjoint clusters in one graph of all possible patient states, a sequence of patient states connected by doctor agents.

$$
G=\left\{\left\langle s_{\text {before }}, a, s_{\text {after }}\right\rangle\right\}
$$

Some states in this oriented graph are very similar, it is reasonable to assume that two different patients have been in the same state at some point. Our algorithm is based on the idea that if two patients have been in the same state, their clinical histories represent two possible timelines of events after the state and the choice of timeline depends on doctor's actions.

We find all state pairs $\left\langle s_{A}, s_{B}\right\rangle$ below a similarity threshold

$$
\cos \left(s_{A}, s_{B}\right)<c_{\min }
$$

and merge each into a single state, replacing occurences of $s_{A}$ and $s_{B}$ in $G$ with $\frac{s_{A}+s_{B}}{2}$. The resulting oriented graph becomes the backbone of out simulator. The simulated patient is initialized in state $s_{0}$ equal to one of the initial states of real patients in MIMIC. When at timestep $t$ the agent picks action $a_{t}$, transition to the next state depends on euclidean distance between the action in the graph and $a_{t}$ via the softmax function:

$$
p\left(s_{t+1} \mid s_{t}, a_{t}\right)=\frac{\sum_{\left\langle s_{\text {before }}, a, s_{\text {after }}\right\rangle}^{G} \mathbb{I}\left[s_{\text {before }}=s_{t}\right] \mathbb{I}\left[s_{\text {after }}=s_{t+1}\right] e^{\left|a_{t}-a\right|_{2}^{2}}}{\sum_{\left\langle s_{\text {before }}, a, s_{\text {after }}\right\rangle} \mathbb{I}\left[s_{\text {before }}=s_{t}\right] e^{\left|a_{t}-a\right|_{2}^{2}}}
$$

where $\mathbb{I}$ is the indicator function. See Figure $\mathbf{8}$ for a diagram of the resulting simulator.

The simulator's source code will be published soon.

\section{EFFECTIVENESS}

How do these simulators fare with respect to accuracy criteria we set out in the introduction? The factors that contribute to a 


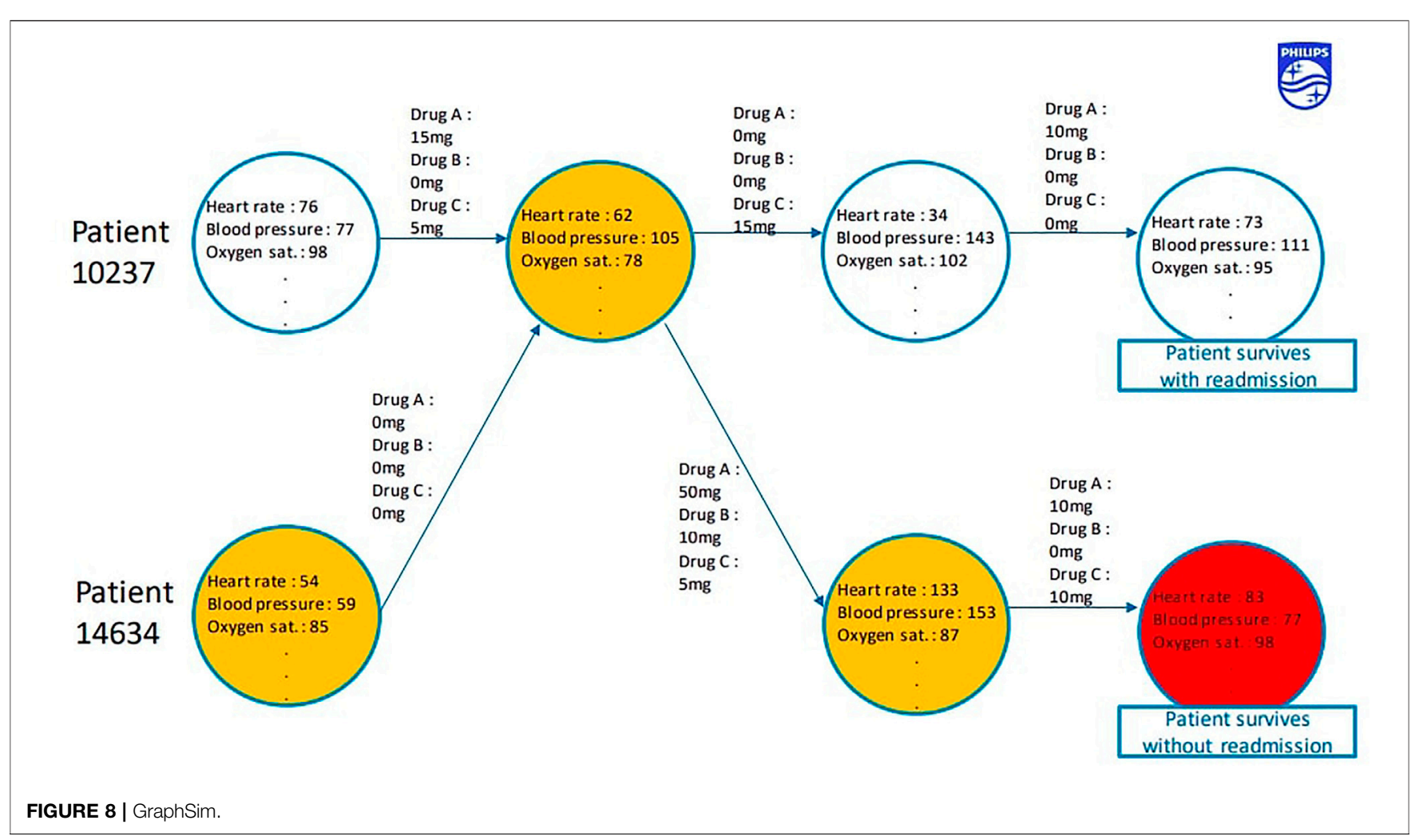

TABLE 2 | Summary of each simulator: trust view.

\begin{tabular}{|c|c|c|c|c|c|}
\hline Simulator & Scope & Data source & $\begin{array}{l}\text { Sample } \\
\text { size }\end{array}$ & Learning algorithm & Known biases \\
\hline simglucose (Xie, 2018) & type 1 diabetes & original study & 32 & expert model validated on data & Overfitting confirmation bias confirmation \\
\hline $\begin{array}{l}\text { GYMIC (Kiani et al., } \\
\text { 2019) }\end{array}$ & $\begin{array}{l}\text { sepsis in intensive } \\
\text { care }\end{array}$ & $\begin{array}{l}\text { MIMIC (Johnson et al., } \\
\text { 2021) }\end{array}$ & 40,000 & behavior cloning & bias no factual basis \\
\hline $\begin{array}{l}\text { Virtu-ALS (Brisk et al., } \\
\text { 2018) }\end{array}$ & emergency care & & & & \\
\hline Auto-ALS & emergency care & & & & \\
\hline HeartPole & healthy lifestyle & & & & \\
\hline GraphSim & intensive care & $\begin{array}{l}\text { MIMIC (Johnson et al., } \\
\text { 2021) }\end{array}$ & 40,000 & graph compression & \\
\hline
\end{tabular}

simulator's accuracy are reviewed in Table 2. GYMIC and GraphSim are the only simulators trained on a large dataset and GYMIC's accurracy has known overfitting issues. GraphSim is thus the most accurate of the simulators.

The most transparent simulator is clearly HeartPole. It does not aim to model any real clinical scenario accurately, but it can be a useful development tool to help scrutinize reinforcement learning algorithms.

As far as difficulty is concerned, HeartPole (Härmä et al., 2021), simglucose (Zhu et al., 2021), and GYMIC (Kiani et al., 2019) are known to be solvable with relatively small models and standard reinforcement learning algorithms like DQN (Mnih et al., 2015). Thus, the only simulators difficult enough to be benchmarks for novel approaches are Virtu-ALS and Auto-ALS and Auto-ALS is the more accessible of the two.
TABLE 3 | Summary of each simulator: POMDP view.

\begin{tabular}{lccc} 
Simulator & $\mathbf{0}$ & $\mathbf{A}$ & $\boldsymbol{p}_{\boldsymbol{r}}(\boldsymbol{r}=\mathbf{0} \mid \mathbf{s}) \neq \mathbf{1}$ \\
\hline simglucose & {$[0 ;+\infty)$} & {$[0 ; 35]$} & $S_{n t} \cup S_{t}$ \\
GYMIC & {$[0 ; 24]^{46}$} & $0, \ldots, 24$ & $S_{n t} \cup S_{t}$ \\
Virtu-ALS & {$[0 ; 256]^{307} 200$} & $1, \ldots, 307,200$ & $S_{n t} \cup S_{t}$ \\
Auto-ALS & {$[0 ;+\infty)^{36}$} & $1, \ldots, 34$ & $S_{n t} \cup S_{t}$ \\
HeartPole & $\mathcal{R}^{6}$ & $1, \ldots, 4$ & $S_{n t} \cup S_{t}$ \\
GraphSim & $(-\infty ;+\infty)^{26}$ & {$[0 ; 1]^{317}$} & $S_{t}$
\end{tabular}

Table 3 reviews the structural complexity of the simulators, a factor that directly contributes to difficulty. Note that Virtu$A L S$ is an unusually high-dimensional environment. As such, 
solving it is likely to require more parameters and longer training times. GraphSim is the only simulator that doesn't provide non-zero rewards in non-terminal states $S_{n t}$, making it harder for the agent to attribute the results of the episode to particular actions. GYMIC (see section 2.2) solves this problem with an additional metric (SOFA score), but unlike GYMIC GraphSim covers a wide range of clinical conditions and there is no single health metric applicable to each.

\section{CONCLUSION}

Automatic discovery of clinical strategies is a nascent field of research that has a potential to considerably improve patient outcomes and become a new modus operandi in healthcare research. The goal of this paper is to provide a solid foundation for further development of this field with better patient simulators and better understanding thereof. We have reviewed the state of the art in patient simulators, identified some of the problems the field is facing and proposed novel simulators to address them. We believe that HeartPole and Auto-ALS can become new standard benchmarks for reinforcement learning in healthcare, while GraphSim can become a stepping stone to improved patient outcomes in intensive care.

\section{REFERENCES}

American Heart Association Staff (2006). Advanced Cardiovascular Life Support Provider Manual (International English). American Heart Association, Incorporated. ISBN 9781616690472. URL Available at: https://books.google. nl/books?id=oxdAygEACAAJ.

Barto, A. G., Sutton, R. S., and Anderson, C. W. (1983). Neuronlike Adaptive Elements that Can Solve Difficult Learning Control Problems. IEEE Trans. Syst. Man. Cybern. SMC-13 (5), 834-846. ISSN 2168-2909. doi:10.1109/ TSMC.1983.6313077

Brisk, R., Bond, R. B., Liu, J. Y., Finlay, D., McLaughlin, J. A. D., and McEneaney, D. (2018). Ai to Enhance Interactive Simulation-Based Training in Resuscitation Medicine. In British HCI Conference 2018, BHCI2018 ; Conference date: 0207-2018 Through 06-07-2018. 1-4. doi:10.14236/ewic/HCI2018.64

Cavalcante, R. C., Brasileiro, R. C., Souza, V. L. F., Nobrega, J. P., and Oliveira, A. L. I. (2016). Computational Intelligence and Financial Markets: A Survey and Future Directions. Expert Syst. Appl. 55, 194-211. doi:10.1016/ j.eswa.2016.02.006

Chen, R. J., Lu, M. Y., Chen, T. Y., Williamson, D. F. K., and Mahmood, F. (2021). Synthetic Data in Machine Learning for Medicine and Healthcare. Nat. Biomed. Eng., 1-5. doi:10.1038/s41551-021-00751-8

Clarke, W., and Kovatchev, B. (2009). Statistical Tools to Analyze Continuous Glucose Monitor Data. Diabetes Technol. Ther. 11 Suppl 1 (S1), S45-S54. doi:10.1089/dia.2008.0138

Gong, M., Xie, Y., Pan, K., Feng, K., and Qin, A. K. (2020). A Survey on Differentially Private Machine Learning [review Article]. IEEE Comput. Intell. Mag. 15 (2), 49-64. doi:10.1109/MCI.2020.2976185

Gulwani, S., Polozov, O., and Singh, R. (2017). Program Synthesis. FNT in Programming Languages 4 (1-2), 1-119. ISSN 2325-1107. doi:10.1561/ 2500000010

Härmä, A., Petković, M., Liventsev, V., and Simon, A. (2021). Heartpole: A Transparent Task for Reinforcement Learning in Healthcare. URL Available at: https://github.com/vadim0x60/heartpole/blob/master/HeartPole_ abstract.pdf.

Hester, R. L., Brown, A. J., Husband, L., Iliescu, R., Pruett, D., Summers, R., et al. (2011). Hummod: A Modeling Environment for the Simulation of Integrative

\section{DATA AVAILABILITY STATEMENT}

Publicly available datasets were analyzed in this study. This data can be found here: https://physionet.org/content/mimiciv/0.4/

\section{AUTHOR CONTRIBUTIONS}

All authors listed have made a substantial, direct, and intellectual contribution to the work and approved it for publication.

\section{FUNDING}

This work was funded by the European Union's Horizon 2020 research and innovation programme under grant agreement $\mathrm{n}^{\circ} 812882$. This work is part of "Personal Health Interfaces Leveraging HUman-MAchine Natural interactionS" (PhilHumans) project: https://www.philhumans.eu

\section{ACKNOWLEDGMENTS}

We would like to thank Rob Brisk of (Brisk et al., 2018) for providing us with Virtu-ALS source code as well as his advice and support.

Human Physiology. Front. Physio. 2 (12). ISSN 1664-042X. doi:10.3389/ fphys.2011.00012

Hochreiter, S., and Schmidhuber, J. (1997). Long Short-Term Memory. Neural Comput. 9 (8), 1735-1780. doi:10.1162/neco.1997.9.8.1735

James, J., Yoon, J., and Van Der Schaar, M. (2018). Pate-gan: Generating Synthetic Data with Differential Privacy Guarantees. In International conference on learning representations.

Jiang, W. (2021). Applications of Deep Learning in Stock Market Prediction: Recent Progress. Expert Syst. Appl. 184, 115537. doi:10.1016/ j.eswa.2021.115537

Johnson, A., Bulgarelli, L., Pollard, T., Horng, S., Celi, L. A., and Mark, R. (2021) Mimic-iv (version 0.4)

Jozefowicz, R., Vinyals, O., Schuster, M., Shazeer, N., and Wu, Y. (2016). Exploring the Limits of Language Modeling. arXiv preprint arXiv:1602.02410.

Kiani, A., Wang, C., and Xu, A. (2019). Sepsis World Model: A Mimic-Based Openai Gym" World Model" Simulator for Sepsis Treatment. arXiv preprint arXiv:1912.07127.

Koza, John. R. (1994). Genetic Programming II, 17. MA: MIT press Cambridge.

Krishnan, R. G., Shalit, U., and Sontag, D. (2015). Deep Kalman Filters. arXiv preprint arXiv:1511.05121.

Lambden, S., Laterre, P. F., Levy, M. M., and Francois, B. (2019). The SOFA ScoreDevelopment, Utility and Challenges of Accurate Assessment in Clinical Trials. Crit. Care 23 (1), 374-379. doi:10.1186/s13054-019-2663-7

Lee, J., Kim, H., Kim, K. H., Jung, D., Jowsey, T., and Webster, C. S. (2020). Effective Virtual Patient Simulators for Medical Communication Training: a Systematic Review. Med. Educ. 54 (9), 786-795. doi:10.1111/medu.14152

Li Deng, Li. (2012). The Mnist Database of Handwritten Digit Images for Machine Learning Research [best of the Web]. IEEE Signal. Process. Mag. 29 (6), 141-142. doi:10.1109/msp.2012.2211477

Liventsev, V., Aki, H., and Petković, M. (2021). Neurogenetic Programming Framework for Explainable Reinforcement Learning. doi:10.1145/3449726.3459537

Liventsev, V. (2021). Auto-als-v0. URL Available at: https:/github.com/ vadim0x60/auto-als.

Liventsev, V. (2021). Anthropodidactic Learning: a Modest Proposal. URL Available at: https://vadim.me/posts/anthropodidactic/.

Liventsev, V. (2021). Reinforcement Learning as Message Passing. URL Available at: https://vadim.me/posts/mpdp/. 
Man, C. D., Micheletto, F., Lv, D., Breton, M., Kovatchev, B., and Cobelli, C. (2014). The UVA/PADOVA Type 1 Diabetes Simulator. J. Diabetes Sci. Technol. 8 (1), 26-34. ISSN 1932-2968. doi:10.1177/1932296813514502

McFetrich, J. (2006). A Structured Literature Review on the Use of High Fidelity Patient Simulators for Teaching in Emergency Medicine. Emerg. Med. J. 23 (7), 509-511. doi:10.1136/emj.2005.030544

Mischel, W., and EbbesenEbbesen, E. B. (1970). Attention in Delay of Gratification. J. Personal. Soc. Psychol. 16 (2), 329-337. doi:10.1037/h0029815

Mnih, V., Kavukcuoglu, K., Silver, D., Graves, A., Antonoglou, I., Wierstra, D., et al. (2013). Playing Atari with Deep Reinforcement Learning. arXiv preprint arXiv: 1312.5602 .

Mnih, V., Kavukcuoglu, K., Silver, D., Rusu, A. A., Veness, J., Bellemare, M. G., et al. (2015). Human-level Control through Deep Reinforcement Learning. nature 518 (7540), 529-533. doi:10.1038/nature14236

Moore, A. W. (1990). Efficient Memory-Based Learning for Robot Control. Cambridge, UK: Technical report.

MühlbacherMühlbacher, A. C., and Juhnke, C. (2013). Patient Preferences versus Physicians' Judgement: Does it Make a Difference in Healthcare Decision Making? Appl. Health Econ. Health Pol. 11 (3), 163-180. doi:10.1007/s40258013-0023-3

Nehring, W. M., and Lashley, F. R. (2004). Current Use and Opinions Regarding Human Patient Simulators in Nursing Education: An International Survey. Nurs. Educ. Perspect. 25 (5), 244-248.

Niazkar, M., and Niazkar, H. R. (2020). Covid-19 Outbreak: Application of MultiGene Genetic Programming to Country-Based Prediction Models. Electron. J. Gen. Med. 17 (5), 2020.

Price, W. N. (2018). Big Data and Black-Box Medical Algorithms. Sci. Transl. Med. 10 (471), eaao5333. doi:10.1126/scitranslmed.aao5333

Raghu, A., Komorowski, M., Ahmed, I., Celi, L., Peter, S., and Ghassemi, M. (2017). Deep Reinforcement Learning for Sepsis Treatment. arXiv preprint arXiv: 1711.09602

Rankin, D., Black, M., Bond, R., Wallace, J., Mulvenna, M., and Epelde, G. (2020). Reliability of Supervised Machine Learning Using Synthetic Data in Health Care: Model to Preserve Privacy for Data Sharing. JMIR Med. Inform. 8 (7), e18910. doi: $10.2196 / 18910$

Street, R. L., Elwyn, G., and Epstein, R. M. (2012). Patient Preferences and Healthcare Outcomes: an Ecological Perspective. Expert Rev. Pharmacoeconomics Outcomes Res. 12 (2), 167-180. doi:10.1586/erp.12.3

Summers, R. L., Ward, K. R., Witten, T., Convertino, V. A., Ryan, K. L., Coleman, T. G., et al. (2009). Validation of a Computational Platform for the Analysis of the Physiologic Mechanisms of a Human Experimental Model of Hemorrhage. Resuscitation 80 (12), 1405-1410. doi:10.1016/ j.resuscitation.2009.09.001
Sutton, R. S., and Barto, A. G. (2018). Reinforcement Learning: An Introduction. MIT press.

Thim, T., Krarup, N. H., Grove, E., Rohde, C., and Lofgren, B. (2012). Initial Assessment and Treatment with the Airway, Breathing, Circulation, Disability, Exposure (Abcde) Approach. Ijgm 5, 117. doi:10.2147/ijgm.s28478

Walonoski, J., Kramer, M., Nichols, J., Quina, A., Moesel, C., Hall, D., et al. (2018). Synthea: An Approach, Method, and Software Mechanism for Generating Synthetic Patients and the Synthetic Electronic Health Care Record. J. Am. Med. Inform. Assoc. 25 (3), 230-238. doi:10.1093/jamia/ocx079

Wang, Z., Myles, P., and Tucker, A. (2021). Generating and Evaluating Crosssectional Synthetic Electronic Healthcare Data: Preserving Data Utility and Patient Privacy. Comput. Intelligence 37 (2), 819-851. doi:10.1111/coin.12427

Watson, D. S., Krutzinna, J., Bruce, I. N., Griffiths, C. E., McInnes, I. B., Barnes, M. R., et al. (2019). Clinical Applications of Machine Learning Algorithms: beyond the Black Box. BMJ 364, 1886, 2019 . ISSN 0959-8138. doi:10.1136/bmj.1886

Wendy, M. N., Ellis, W. E., and Lashley, F. R. (2001). Human Patient Simulators in Nursing Education: An Overview. Simulation \& Gaming 32 (2), 194-204.

Xie, J. (2018). Simglucose V0. 2.1, 2018 URL Available at: https:/github.com/ jxx123/simglucose.

Yu, C., Liu, J., and Nemati, S. (2019). Reinforcement Learning in Healthcare: A Survey. CoRR. abs/1908.08796URL Available at: http://arxiv.org/abs/1908. 08796.

Zhu, T., Li, K., and Georgiou, P. (2021). Personalized Dual-Hormone Control for Type 1 Diabetes Using Deep Reinforcement Learning. Cham: Springer International Publishing, 45-53. doi:10.1007/978-3-030-53352-6_5

Conflict of Interest: The authors declare that the research was conducted in the absence of any commercial or financial relationships that could be construed as a potential conflict of interest.

Publisher's Note: All claims expressed in this article are solely those of the authors and do not necessarily represent those of their affiliated organizations, or those of the publisher, the editors and the reviewers. Any product that may be evaluated in this article, or claim that may be made by its manufacturer, is not guaranteed or endorsed by the publisher.

Copyright (c) 2021 Liventsev, Härmä and Petković. This is an open-access article distributed under the terms of the Creative Commons Attribution License (CC BY). The use, distribution or reproduction in other forums is permitted, provided the original author(s) and the copyright owner(s) are credited and that the original publication in this journal is cited, in accordance with accepted academic practice. No use, distribution or reproduction is permitted which does not comply with these terms. 